\title{
Correlação dos fatores sociodemográficos e clínicos com alterações da funcionalidade em crianças hospitalizadas
}

\author{
Correlation of sociodemographic and clinical \\ factors with changes in functionality in \\ hospitalized children
}

\section{Lene Gomes de Jesus ${ }^{1}$ (c) Maria Carolina de Britto Andrade 2}

${ }^{1}$ Autora para correspondência. Hospital Geral Roberto Santos (Salvador). Bahia, Brasil. lenegomesvieira@hotmail.com ${ }^{2}$ Hospital Geral Roberto Santos (Salvador). Bahia, Brasil. carolbritto27@hotmail.com

RESUMO | INTRODUÇÃO: O desenvolvimento de alterações funcionais é comum em crianças que estiveram em condições de terapia intensiva. A Functional Status Scale (FSS) é uma escala que tem por objetivo avaliar a funcionalidade de pacientes pediátricos hospitalizados. OBJETIVO: Verificar a possível correlação de fatores sociodemográficos e clínicos com as alterações funcionais de crianças submetidas à internação na UTIP. MATERIAIS E MÉTODOS: Estudo coorte com crianças admitidas em uma UTIP. A funcionalidade foi avaliada pelo FSS na admissão e na alta da unidade. Nova incapacidade foi definida como mudança no FSS $\geq 1$ e incapacidade grave como mudança no FSS $\geq 3$. Os dados foram avaliados pelo programa SPSS versão 17.0. O teste de Pearson e Spearman foi empregado para análise de correlação. O nível de significância estatística adotada foi de $p<0,05$. RESULTADOS: Participaram do estudo 43 crianças, sendo $51,2 \%$ do sexo masculino, com mediana de idade de 15 (1-144) meses. O principal motivo de internação foi desconforto respiratório $12(27,9 \%)$ e a comorbidade prévia mais frequente foi a neurológica (37,3\%), seguida de cardiovascular (11,8\%). Foram observadas correlações significativas entre nova incapacidade e doença vascular prévia $(p<0,000)$; nova incapacidade e internamento por alteração vascular $(p<0,000)$ e incapacidade grave e doença cardiovascular prévia $(p=0,005)$. CONCLUSÃO: $O$ desenvolvimento de alterações funcionais pode ocorrer em crianças após internação na UTIP. A presença prévia de doença vascular e doença cardíaca, além do internamento por alteração vascular, estão associados a uma piora funcional na alta hospitalar.

PALAVRAS-CHAVE: Unidade de Terapia Intensiva Pediátrica. Criança hospitalizada. Desempenho funcional. Morbidade.

\begin{abstract}
INTRODUCTION: The development of functional changes is common in children who have been in intensive care conditions. The Functional Status Scale (FSS) is a scale that aims to assess the functionality of hospitalized pediatric patients. OBJECTIVE: To verify the correlation of sociodemographic and clinical factors with the functional changes of children submitted to PICU admission. MATERIALS AND METHODS: A cohort study with children admitted to a PICU. The FSS assessed functionality on admission and discharge from the unit. New disability was defined as a change in FSS $\geq 1$ and severe disability as a FSS $\geq$ 3. Data were evaluated using the SPSS version 17.0 program. Pearson and Spearman's test was used for correlation analysis. The level of statistical significance adopted was $p$ $<0.05$. RESULTS: 43 children participated in the study; $51.2 \%$ were male, with a median age of 15 (1-144) months. The main reason for hospitalization was respiratory discomfort $12(27.9 \%)$, and the most frequent previous comorbidity was neurological (37.3\%), followed by cardiovascular (11.8\%). Significant correlations were observed between new disability and previous vascular disease $(p<0.000)$, new disability and hospitalization due to vascular changes ( $p<0.000$ ), and severe disability and previous cardiovascular disease $(p=0.005)$. CONCLUSION: The development of functional changes can occur in children after admission to the PICU. The previous presence of vascular disease and heart disease, and hospitalization due to vascular changes are associated with a functional worsening at hospital discharge.
\end{abstract}

KEYWORDS: Intensive care units pediatric. Child hospitalized. Functional performance. Morbidity. 


\section{Introdução}

A assistência nas Unidades de Terapia Intensiva Pediátrica (UTIP) vem sofrendo diversas transformações ao longo dos anos. ${ }^{1-3}$ Com os avanços tecnológicos e a melhoria da assistência, houve uma redução nas taxas de mortalidade na maioria das UTIP, mas, em contrapartida, estudos recentes demonstram aumento das taxas de morbidade após internação nessas unidades. ${ }^{1-3}$ Devido a essas mudanças, além da avaliação da sobrevida, é crescente o interesse dos pesquisadores por desfechos funcionais após internação na UTI. ${ }^{2.3}$ Frente a essas informações, sabe-se que o objetivo das equipes assistenciais nas UTIP não se restringe a salvar vidas, mas também executar ações de prevenção de morbidades e preservação da função. ${ }^{2}$

O hospital é um ambiente que oferece certa privação aos estímulos fundamentais para o desenvolvimento infantil. $4,5 \mathrm{~A}$ internação hospitalar prolongada geralmente leva à redução da funcionalidade, e esta alteração pode estar relacionada à doença de base ou associada a outros fatores, como aos cuidados administrados nas UTIP. ${ }^{4,5} \mathrm{~A}$ literatura relata que o declínio da funcionalidade, incluindo déficits físicos, psicossociais e neuro-cognitivos, é comum em crianças que estiveram em condições de terapia intensiva. ${ }^{2-4}$ As incapacidades e limitações apresentada pelas crianças após alta da UTIP afetam o seu desempenho e o desenvolvimento global, seja na dimensão física, cognitiva, emocional e/ou social.., 5

Sendo assim, é de grande importância a utilização de instrumentos que avaliem a funcionalidade de crianças hospitalizadas. ${ }^{6-8} \mathrm{~A}$ aplicação desses instrumentos permite identificar precocemente as crianças mais propensas a desenvolver alterações na funcionalidade, o que favorece estratégias mais consistentes de tratamento e reabilitação.,., 7 No Brasil, grande parte desses instrumentos ainda não estão disponíveis, pois não são validados para a língua portuguesa.

Recentemente, um instrumento especifico para avaliar a funcionalidade, a Functional Status Scale(FSS), passou pelo processo de validação na população brasileira e desde então tem sido amplamente utilizada. $., 9,10$
Esse instrumento foi desenvolvido com base conceitual em escalas de atividades de vida diária e de comportamento adaptativo, e seu objetivo é avaliar os desfechos funcionais de pacientes pediátricos hospitalizados. $., 9,10$ A FSS é um instrumento apropriado para uma ampla faixa etária, considerado de fácil aplicação, baixo custo e confiável. $., 8,9$

Dada a importância da melhor compreensão das alterações de funcionalidade durante o processo de hospitalização da criança, este estudo teve por objetivo verificar a correlação dos fatores sociodemográficos e clínicos com as alterações funcionais, medidas pelo FSS, de crianças submetidas à internação na UTIP.

\section{Materiais e métodos}

Trata-se de um estudo observacional, do tipo coorte prospectivo, cuja população foi composta por crianças admitidas em uma UTIP. Foram incluídas no estudo crianças de ambos os sexos, com idades entre 29 dias e 15 anos de vida, em ventilação espontânea e que não estivessem sob o uso contínuo de sedativos ou anticonvulsivantes em doses além das habituais, e excluídas do estudo as crianças readmitidas na UTIP em um período $\leq 24$ horas após a alta da unidade. A amostra foi constituída por conveniência.

Dados primários, relacionados ao aspecto funcional, foram coletados por meio da aplicação do formulário de investigação, e dados secundários, sociodemográficos e clínicos foram provenientes dos prontuários dos pacientes. A coleta de dados foi realizada na UTIP entre os meses de fevereiro a abril de 2020 por fisioterapeutas do serviço, previamente treinados. No momento da admissão na UTIP, a pesquisa era apresentada ao responsável e em caso de concordância com os propósitos do estudo e após assinar o Termo de Consentimento Livre e Esclarecido (TCLE), era preenchido um formulário com dados basais provenientes do prontuário. Na sequência, era aplicada a FSS para identificar o nível de funcionalidade da criança no momento da admissão na UTIP. A FSS também foi aplicada na alta da unidade. 
Como variáveis sociodemográficas, foram incluídas o sexo, categorizado em feminino e masculino, e idade em meses. As variáveis clínicas dizem respeito às comorbidades prévias, descritas em: doença neurológica, cardíaca, renal, vascular, cirurgia abdominal prévia e múltiplas comorbidades (na presença de duas ou mais comorbidades) e o motivo da internação na UTIP como desconforto respiratório, pós-operatório de cirurgia pediátrica geral, pós operatório de neurocirurgia, rebaixamento do nível de consciência e alterações vasculares.

A FSS foi desenvolvida para avaliar o estado funcional de pacientes hospitalizados, é composto por seis domínios: estado mental, funcionamento sensorial, comunicação, funcionamento motor, alimentação e estado respiratório, a pontuação por domínio vai de 1 (normal) a 5 (disfunção muito grave). Portanto, seu escore global pode variar de 6 a 30 pontos, quanto maior a pontuação pior a funcionalidade. O escore global ainda pode ser categorizado da seguinte forma: funcionalidade adequada, nas pontuações que vão de 6 a 7; disfunção leve de 8 a 9 pontos; disfunção moderada entre 10 e 15 pontos; disfunção grave de 16 a 21 pontos e acima de 21 será considerado disfunção muito grave ${ }^{6,9,10}$. De acordo com o estudo de Williams e colaboradores 11, nova incapacidade foi definida como uma mudança no FSS $\geq 1$ e incapacidade grave como uma mudança no FSS $\geq 3$, esta variável teve por objetivo mostrar os indivíduos que tiveram alteração funcional pelo FSS, no que diz respeito a pontuação da admissão e da alta hospitalar.

O programa Statistical Package for the Social Sciences (SPSS) versão 17.0, foi utilizado para estruturar a planilha de dados e a análise estatística. As variáveis numéricas foram apresentadas em mediana e intervalo interquartil e as variáveis categóricas apresentadas em termos absolutos e frequência relativa. Para este estudo foi realizada uma correlação de Pearson entre as variáveis sociodemograficas e clinicas com as alterações funcionais avaliada pelo FSS. $0 p<0,05$ foi considerado estatisticamente significante.

O presente estudo faz parte de um projeto-mãe e encontra-se aprovado pelo Comitê de Ética e Pesquisa.
Segundo a Resolução 466/12 de pesquisa envolvendo seres humanos, todos os participantes ou familiar responsável assinaram o TCLE.

\section{Resultados}

Participaram do estudo 43 crianças internadas na UTIP na cidade de Salvador, Bahia, sendo $51,2 \%$ do sexo masculino, com mediana de idade de 15 (1-144) meses. A mediana do tempo de internação foi de 6 (1-27) dias. Com relação aos principais motivos de internação, 12 (27,9\%) participantes apresentaram desconforto respiratório, $8(18,6 \%)$ pós operatório de cirurgia pediátrica e 6 (14\%) pós operatório de neurocirurgia. A comorbidade prévia mais frequente foi doença neurológica $(37,3 \%)$, seguida de doença cardíaca $(11,8 \%)$ e renal $(9,8 \%)$ (Tabela 1$)$.

Com relação ao escore global da FSS, na admissão, a amostra apresentou uma mediana de 14 (6-26) pontos, indicando então disfunção moderada. Já na alta hospitalar, a mediana do escore global reduziu para 9 (6-24) pontos, caracterizando a amostra como disfunção leve (Tabela 1). Na admissão, os pesquisadores evidenciaram que a maioria dos participantes apresentavam disfunção grave 14 (32,6\%), em contra partida, no momento da alta hospitalar, a maioria 19 $(44,2 \%)$ apresentava funcionalidade adequada, mostrando assim um aumento do número de crianças com melhora da funcionalidade na alta da unidade (Tabela 2). No que diz respeito às alterações funcionais, o presente estudo demonstrou que 9,3\% das crianças tiveram uma piora funcional na alta, sendo que dentre esses, 1 (2,3\%) apresentou nova incapacidade (mudança no FSS $\geq 1$ ) e 3 (7\%) tiveram incapacidade grave (mudança no FSS $\geq 3$ ) (Tabela 1).

Ao correlacionar as variáveis sociodemográficas e clínicas com as alterações funcionais, foram observadas associações significativas entre nova incapacidade e doença vascular prévia $(p<0,000)$; nova incapacidade e internamento por alteração vascular $(p<0,000)$; e incapacidade grave e doença cardiovascular prévia $(p=0,005)$ (Tabela 3). 
Tabela 1. Características sociodemográficas, clínicas e funcionais das crianças internadas em uma UTIP, 2020

\begin{tabular}{|c|c|}
\hline Variáveis & $\begin{array}{l}\text { População total } \\
\qquad(n=43)\end{array}$ \\
\hline $\begin{array}{l}\text { Sexo masculino, n (\%) } \\
\text { Idade (meses), mediana (IQ) }\end{array}$ & $\begin{array}{l}22(51,2) \\
15(1-144)\end{array}$ \\
\hline Tempo de internação na UTI (dias), mediana (IQ) & $6(1-27)$ \\
\hline \multicolumn{2}{|l|}{ Motivo da internação, n (\%) } \\
\hline Desconforto respiratório & $12(27,9)$ \\
\hline Pós operatório de cirurgia pediátrica & $8(18,6)$ \\
\hline Pós operatório de neurocirurgia & $6(14)$ \\
\hline Rebaixamento do Nível de consciência & $5(11,6)$ \\
\hline Choque e sepse & $4(9,3)$ \\
\hline Alterações vasculares & $2(4,7)$ \\
\hline Outros & $6(14)$ \\
\hline \multicolumn{2}{|l|}{ Comorbidades prévia, n (\%) } \\
\hline Doença Neurológica & $19(37,3)$ \\
\hline Doença Cardíaca & $6(11,8)$ \\
\hline Doença Renal & $5(9,8)$ \\
\hline Doença Vascular & $3(5,9)$ \\
\hline Cirurgia abdominal prévia & $2(3,9)$ \\
\hline Doença Respiratória & $1(2)$ \\
\hline Outras & $4(7,8)$ \\
\hline Múltiplas comorbidades & $4(7,8)$ \\
\hline Sem comorbidade prévia & $7(13,7)$ \\
\hline Escore global da FSS na admissão, mediana (IQ) & $14(6-26)$ \\
\hline Escore global da FSS na alta, mediana (IQ) & $9(6-24)$ \\
\hline Alterações funcionais (mudança no FSS), $n$ (\%) & $4(9,3)$ \\
\hline$\geq 1$ & $1(2,3)$ \\
\hline$\geq 3$ & $3(7)$ \\
\hline
\end{tabular}

Tabela 2. Classificação da FSS na admissão e na alta de uma UTIP , 2020

\begin{tabular}{lcc}
\hline Classificação da FSS & $\begin{array}{c}\text { Admissão } \\
(\mathrm{n}=43)\end{array}$ & $\begin{array}{c}\text { Alta } \\
(\mathrm{n}=43)\end{array}$ \\
\hline 6-7 Funcionalidade adequada, $\mathrm{n}(\%)$ & $6(14)$ & $19(44,2)$ \\
8-9 disfunção leve, $\mathrm{n}(\%)$ & $8(18,6)$ & $3(7)$ \\
$10-15$ disfunção moderada, $\mathrm{n}(\%)$ & $11(25,6)$ & $11(25,6)$ \\
$16-21$ disfunção grave, $\mathrm{n}(\%)$ & $14(32,6)$ & $8(18,6)$ \\
$>21$ disfunção muito grave, $n(\%)$ & $4(9,3)$ & $2(4,7)$ \\
\hline
\end{tabular}

Tabela 3. Correlação entre as variáveis sociodemográficas e clínicas com as alterações funcionais das crianças internadas em uma UTIP, 2020

\begin{tabular}{l|lc}
\hline \multicolumn{1}{c}{ Variáveis } & p-valor \\
\hline \multirow{2}{*}{ Nova incapacidade } & Doença vascular prévia & $p<0,000$ \\
\cline { 2 - 3 } & Internamento por alteração vascular & $p<0,000$ \\
\hline \multirow{2}{*}{ Incapacidade grave } & Doença cardíaca prévia & $p=0,005$ \\
\hline
\end{tabular}




\section{Discussão}

Os resultados do presente estudo sugerem que o desenvolvimento de nova incapacidade e incapacidade grave pode ocorrer em crianças que passaram por internação em UTI. Resultado semelhante foi encontrado no estudo multicêntrico de Pollack et al. ${ }^{3}$, cujo objetivo foi investigar o desenvolvimento de nova incapacidade (aumento do FSS $\geq 3$ ) associado aos cuidados intensivos pediátricos também através da aplicação da FSS, e a incidência de nova incapacidade foi de $4,8 \%$.

A literatura refere índices ainda maiores de agravos funcionais após alta da UTIP, como o estudo no estudo de Williams e colaboradores ${ }^{11}$, onde a apresentação de nova incapacidade (mudança no FSS $\geq 1$ ) e incapacidade grave (mudança no FSS $\geq 3$ ) foram corriqueiras em pacientes pediátricos com diagnóstico neurológico primário, acometendo 35\% e 13\% da sua população respectivamente, na alta da UTIP. Esta frequência elevada, quando comparado ao quantitativo das alterações funcionais encontradas no presente estudo, pode estar relacionada a diferenças existentes com relação a especialidade da UTIP, ao suporte tecnológico e ao perfil dos pacientes avaliados que se altera de acordo com a especificidade de cada região.

Estudos recentes mostram que algumas variáveis como o motivo da internação na UTIP e a presença de comorbidade prévia podem influenciar significativamente o estado funcional dos pacientes internados nessas unidades. ${ }^{3,10-13}$ Pollack e colaboradores ${ }^{3}$ relataram que a presença de nova incapacidade (FSS $\geq 3$ ) na alta da UTIP, ocorreu de forma mais frequentes em crianças com diagnóstico neurológico primário (7,3\%), doença cardiovascular adquirida $(5,9 \%)$ e doença cardiovascular congênita $(4,9 \%)$, as taxas foram significativamente diferentes entre os grupos $(p=0,003)$. No presente trabalho foi observado resultado semelhante com a literatura, já que foi encontrado associação significativa entre a presença doença vascular prévia, internamento por alteração vascular e o desenvolvimento de nova incapacidade, bem como a presença de doença cardíaca prévia e o desenvolvimento de incapacidade grave. No estudo de Typpo et al..$^{14}$, os pesquisadores encontraram que crianças com doença cardíaca crônica tem maior incidência da síndrome de deficiência de múltiplos órgãos durante internação em UTI quando comparadas a crianças com outras doenças crônicas e a crianças sem doenças crônica, esse achado clínico pode justificar o pior desfecho funcional apresentado por esse perfil de paciente na alta da unidade.

No presente estudo, apesar de a maior parte da amostra apresentar como comorbidade prévias doenças neurológicas, não foi observado associação estatisticamente significativa dessa categoria com o desenvolvimento de nova incapacidade ou incapacidade grave. Isso pode ser explicado pelo estudo de Mestrovic et al. ${ }^{12}$, que teve por objetivo avaliar o desfecho funcional de pacientes tratados em uma UTIP por meio do Pediatric Overall Performance Category. Os pesquisadores constataram que crianças com alterações neurodesenvolvimentais prévias apresentaram escore funcional pré-admissão significativamente pior e por tanto não sofrem tanta deterioração funcional durante a internação hospitalar quando comparadas com crianças sem doenças crônicas e com crianças com outras doenças crônicas não tão graves.

Com relação ao tempo de internação na UTIP, a literatura relata que esta variável apresenta correlação significativa com as perdas funcionais no momento da alta, indicando que quanto maior o período de exposição a esse ambiente, possivelmente, maiores serão as perdas funcionais., 10-13 A mediana de dias de internação encontrada no presente trabalho é semelhante ao referido no estudo de Danneberg $\frac{13}{}$, que por sua vez descreveu uma mediana de 5 (3-10) dias, além de encontrar correlação significativa desta variável com perdas funcionais na alta da unidade em todos os pacientes $(p=0,000)$. Apesar desse achado, no presente estudo, não foi observado correlação significativa do tempo de internação com alterações de funcionalidade. A idade da criança também é apontada como um fator que interfere na funcionalidade, Pollack et al. $\underline{3}$ relataram presença de nova incapacidade em todas as categorias de idade, sendo mais frequente nos menores de 12 meses, com diferença estatisticamente significativa entre os grupos $(p<0,0001)$. Não encontramos associação entre nova incapacidade e idade, isso pode ter ocorrido devido ao pequeno número de participantes. 
Por fim, os resultados do presente estudo sugerem que apesar ocorrer alterações funcionais nesse perfil de crianças, a internação na UTIP não determina piora da funcionalidade, uma vez que houve redução do número de crianças com piora funcional na alta. Este achado difere do encontrado na literatura, que aponta para um pior status funcional no momento da alta da UTIP em comparação com a funcionalidade avaliada pelo FSS na admissão.,13 Este desencontro pode ter ocorrido pois a maioria dos estudos avaliaram o nível funcional da criança prévio a admissão hospitalar, ao contrário do presente trabalho, que avaliou o estado funcional no momento da admissão, onde a criança já poderia apresentar alterações funcionais advindas do quadro agudo que motivou sua internação. Além disso, nos estudos citados, a avaliação e classificação das condições funcionais prévias à admissão na UTIP foi obtida através do relato dos pais e cuidadores podendo conter tendência a supervalorizar as condições das crianças.

Considera-se como limitação do presente estudo a amostra ser proveniente de um único serviço, bem como ser selecionada de forma não-probabilística, podendo não refletir as reais condições de vida e saúde de outras regiões. Além disso, o FSS não foi classificado por domínios, o que permitiria uma avaliação mais detalhada do perfil funcional dessas crianças. Também deve-se levar em consideração o tamanho da amostra e que a associação significativa encontrada no presente estudo com relação à nova incapacidade, representa a condição de apenas uma criança.

\section{Conclusão}

No presente estudo, identificamos que o desenvolvimento de alterações funcionais pode ocorrer em crianças que passaram por internação na UTIP e que existe associação estatisticamente significativa entre nova incapacidade e doença vascular prévia; nova incapacidade e internamento por alteração vascular; e incapacidade grave e doença cardíaca prévia. Identificar e entender as mudanças sutis no FSS é importante para detectar o perfil de pacientes que apresentam risco para desenvolver agravos funcionais, proporcionando assim melhores estratégias de recuperação e reabilitação. Portanto, faz-se necessário desenvolver estudos multicêntricos, com maior número de participantes, priorizando amostragem probabilística, com o objetivo de investigar de forma mais detalhada e a longo prazo as alterações funcionais desenvolvidas pelas crianças durante a hospitalização.

\section{Contribuições das autoras}

Jesus LG participou da coleta de dados, busca e análise estatística dos dados da pesquisa, interpretação dos resultados, redação do artigo científico. Andrade MCB participou da concepção, coleta de dados, interpretação dos resultados e orientação da redação do artigo científico.

\section{Conflitos de interesses}

Nenhum conflito financeiro, legal ou político envolvendo terceiros (governo, empresas e fundações privadas, etc.) foi declarado para nenhum aspecto do trabalho submetido (incluindo, mas não se limitando a subvenções e financiamentos, participação em conselho consultivo, desenho de estudo, preparação de manuscrito, análise estatística, etc.).

\section{Referências}

1. Warwick Butt. Desfecho após alta da unidade de terapia intensiva pediátrica. J. Pediatr. (Rio J.). 2012; 88(1):1-3. https://doi. org/10.2223/JPED.2165

2. Heneghan JA, Pollack MM. Morbidity: Changing the outcome paradigm for pediatric critical care. Pediatr Clin North Am. 2017;64:1147-65. https://doi.org/10.1016/j.pcl.2017.06.011

3. Pollack MM, Holubkov R, Funai T, Clark A, Berger JT, Meert K, et al. Pediatric intensive care outcomes: development of new morbidities during pediatric critical care. Pediatr Crit Care Med. 2014;15(9):821-7. https://doi.org/10.1097/pcc.0000000000000250

\section{Bennett TD. Functional status after pediatric critical care: is it the disease, the cure, or Both? Pediatr Crit Care Med. 2015;16(4):377-8. https://dx.doi. org/10.1097\%2FPCC.0000000000000380}

5. Herrup EA, Wieczorek B, Kudchadkar SR. Characteristics of postintensive care syndrome in survivors of pediatric critical illness: A systematic review. World J Crit Care Med. 2017;6(2):12434. https://dx.doi.org/10.5492\%2Fwjccm.v6.i2.124

\footnotetext{
6. Bastos VCS, Carneiro AAL, Barbosa MSR, Andrade LB. Versão brasileira da Functional Status Scale pediátrica: tradução e adaptação transcultural. Rev. bras. ter. intensiva [Internet]. 2018;30(3):301-7. Disponível em: https://www.scielo.br/pdf/rbti/ v30n3/0103-507X-rbti-20180043.pdf
} 
7. Pollack MM, Holubkov R, Funai T, Clark A, Moler F, Shanley T, et al. Relationship between the functional status scale and the pediatric overall performance category and pediatric cerebral performance category scales. JAMA Pediatr. 2014;168(7):671-6. https://doi.org/10.1001/jamapediatrics.2013.5316

8. Pollack MM, Holubkov R, Glass P, Dean JM, Meert KL, Zimmerman J, et al. Functional Status Scale: new pediatric outcome measure. Pediatrics. 2009;124(1):e18-28. https://doi. org/10.1542/peds.2008-1987

9. Pereira GA, Schaan CW, Ferrari RS, Normann TC, Rosa NV, Ricachinevsky CP, et al. Functional Status Scale: CrossCultural Adaptation and Validation in Brazil. Pediatr Crit Care Med. 2019;20(10):e457-e463. https://doi.org/10.1097/ PCC.0000000000002051

10. Pereira GA, Schaan CW, Ferrari RS. Avaliação funcional em pacientes pediátricos após alta da unidade de terapia intensiva por meio da Functional Status Scale. Rev. bras. ter. intensiva [Internet]. 2017;29(4):460-5. Disponível em: https://www.scielo.br/ pdf/rbti/v29n4/0103-507X-rbti-20170066.pdf
11. Williams CN, Eriksson CO, Kirby A, Piantino JA, Hall TA, Luther $\mathrm{M}$, et al. Hospital Mortality and Functional Outcomes in Pediatric Neurocritical Care. Hosp Pediatr. 2019;9(12):958-66. https://doi. org/10.1542/hpeds.2019-0173

12. Mestrovic J, Polic B, Mestrovic G, Kardum G, Marusis E, Sustic A. Desfecho funcional de crianças tratadas em unidade de terapia intensiva. J. Pediatr. (Rio J.). 2008;84(3):232-6. https://doi. org/10.2223/JPED.1779

13. Danneberg VC. Avaliação de funcionalidade em crianças internadas em unidade de terapia intensiva pediátrica de hospital terciário [dissertação] [Internet]. Porto Alegre: Universidade Federal do Rio Grande do Sul; 2018. Disponível em: https://www. lume.ufrgs.br/handle/10183/188965

14. Typpo KV, Petersen NJ, Petersen LA, Mariscalco MM. Children with chronic illness return to their baseline functional status after organ dysfunction on the first day of admission in the pediatric intensive care unit. J Pediatr. 2010;157(1):108-13.e1. https://doi. org/10.1016/j.jpeds.2009.12.029 